\title{
Application of AI for Analysis of Parkinson's Disease
}

\author{
Harsh Pandey, Arjun Shivnani, Aryaman Chauhan, Aditya Pratap Singh, Pauras Khadakban
}

\begin{abstract}
Parkinson's disease is an issue of the central tactile framework that impacts advancement provoking shudders. The tangible cell is hurt in the frontal cortex causing dopamine levels to drop which prompts the condition. Parkinson's is a reformist ailment that causes degeneration of the frontal cortex, provoking both motor and mental issues. While Dysphonia is a voice issue that causes mandatory fits in the larynx muscle, this is one of its indications. While, Bradykinesia, which is ordinarily described as slowness of improvements, is one of the cardinal signs of Parkinson's sickness (PD). Essential clinical rating scales are used usually to measure bradykinesia in routine clinical practice albeit this kind of examination is uneven. It requires clinical investigation, and it can happen starting from the age of 6. Along these lines, this is a starter study that endeavors to recognize connections between Parkinson's contamination factors for basic unmistakable verification of the sickness. There are 1 million cases in India. It is hence reasonable to acknowledge that there is a connection between a patient's ability to talk/make and the development towards Parkinson's as these limits rot as time propels. The mark of the examination was to survey the features of the sound data and the hour of contorting drawing as an extent of bradykinesia. Henceforth to make strong proof that vocalization data and the handwriting test from a patient can assist with dissecting whether they experience the evil impacts of Parkinson's. As needs be, it is at first anticipated that there is an association between the two. We attempt to run distinctive AI classifiers on the data in wants to show up at a high consistency rate that is facilitated with a reasonable runtime. The dataset managed is procured from a new report by the journal, IEEE Transactions on Biomedical Engineering, of various limits of voice repeat. The actual assessment obtained a consistency speed of $95.58 \%$ hence we want to show up at a rate close to this or possibly to beat it.
\end{abstract}

Keywords: Parkinson's Disease, Machine Learning, Logistic Regression, Extremely Randomized Trees Classifier

Manuscript received on August 03, 2021.

Revised Manuscript received on September 09, 2021.

Manuscript published on September 30, 2021.

* Correspondence Author

Harsh Pandey*, IT Professional, Department of Information Technology, Manipal University Jaipur (Rajasthan), India. Email harshpandeydps@gmail.com

Arjun Shivnani, IT Professional, Department of Information Technology, Manipal University Jaipur (Rajasthan), India. Email: arjunshivnani@gmail.com

Aryaman Chauhan, IT Professional, Department of Information Technology, Manipal University Jaipur (Rajasthan), India. Email: chauhan.aryamaansingh@gmail.com

Aditya Pratap Singh, IT Professional, Department of Information Technology, Manipal University Jaipur (Rajasthan), India. Email: singh.adi2407@gmail.com

Pauras Khadakban, IT Professional, Department of Information Technology, Manipal University Jaipur (Rajasthan), India. Email: paurask1@gmail.com

(c) The Authors. Published by Blue Eyes Intelligence Engineering and Sciences Publication (BEIESP). This is an open access article under the CC BY-NC-ND license (http://creativecommons.org/licenses/by-nc-nd/4.0/)

\section{INTRODUCTION}

$\mathrm{P}$ Parkinson's disorder is an issue of the central tactile framework that impacts advancement provoking tremors. The tangible cell is hurt in the brain causing dopamine levels to drop which prompts the condition. Parkinson's is a reformist sickness that causes degeneration of the brain, inciting both motor and scholarly issues. Parkinson's Disease signs decay as the condition progresses as time goes on. Before all else periods of Parkinson's Disease, one face might show close to zero attitude. Your arms may not swing when you walk. The talk might end up being fragile and slurred. Early investigation and treatment of PD can enough lethargic or end contamination development. In the most skeptical situations, it can in like manner brief Dementia.

Parkinson's symbols and indicators may include:

- Tremor: A tremor, or trembling, generally jerks in a part, once in a while your arms and hands. You may brush your fingers and point forward and in reverse, known as a medication shiver.

- Slowed movement: Some time afterwards, Parkinson's ailment might reasonable your turn of events, making direct tasks problematic and monotonous. Your means might turn out to be more restricted when you walk. It may be difficult to get away from a seat. You might slow down as you endeavor to walk.

- Stiff muscles. Muscle insistence might happen anywhere in the body. The firm influences can be hard and limit scope of measure.

- Impaired posture and balance: Your position might get stooped, or you might have balance issues in view of Parkinson's disease.

- Loss of automatic movements: You might have a reduced ability to perform negligent turns of events, including gleaming, smiling, or swinging your arms when you walk.

- Speech changes: You might talk gently, quickly, slur or postponement preceding talking. Your talk may be even more rambling rather than with the regular inflections.

- Writing changes. Writing gets affected very badly.

The reason for Parkinson's basically stays obscure. Nonetheless, speculations including oxidative harm, ecological poisons, hereditary factors and quickened maturing have been talked about as possible reasons for the illness. In 2005, analysts found a solitary transformation in a Parkinson's infection quality (first distinguished in 1997), which is accepted liable for five percent of acquired cases. Dysphonia is a voice problem that causes compulsory fits in the larynx muscle, this is one of its indications. 


\section{Application of AI for Analysis of Parkinson's Disease}

It is consequently sensible to accept a relationship between a patient's capacity to talk and the movement towards Parkinson's as these capacities fall apart. On account of the diminishing in motor control that is the indication of the sickness, voice can be used to recognize and examine the contamination. With degrees of progress in advancement and the prevalence of sound social occasion devices in step-by-step lives, strong models that can make an understanding of this sound data into an indicative device for clinical consideration specialists would possibly give examine that are more affordable and more exact. We offer evidence to endorse this thought here using a voice dataset assembled from people with and without PD.

Bradykinesia is characterized by sluggish development and an impeded capacity to move the body quickly on order. It is most usually a manifestation of PD or a result of prescriptions. It is one of the fundamental side effects specialists will search for when diagnosing Parkinson's. It is additionally identified with what makes those with Parkinson's lose their capacity to talk unmistakably. Your voice will get gentler and milder over the long run, and your words will turn out to be harder to see plainly. Spiral drawing is a standard neurologic test that is routinely performed anyway sometimes quantified. The twisting assessment is an automated method for taking apart kinematic lead of the upper limb reliant upon twisting drawing on a digitizing tablet that gives practical and strong documents of motor work in a short and easy to control the test. Motor side effects of PD are commonly surveyed utilizing clinical scales, for example, the brought together PD rating scale (UPDRS), which is utilized to assess the presence and seriousness of PD manifestations just as indications changes. Notwithstanding, clinical scales-based estimations can't catch varieties in side effects ceaselessly and they are inhumane toward inconspicuous changes. The determination of Parkinson's is principally founded on the normal side effects laid out above. There is no X-beam or blood test that can affirm the illness to uncover the full degree of the patient's condition and forestall review and detailing inclination, the manifestations should be caught often, just as both when prescription.

\section{A. Medical Treatments:}

For the present, there are no medications open for this neurological contamination, yet medications can assist with controlling your signs, consistently definitely. In some later cases, operation may be urged.

\section{B. Levodopa}

It is frequently viewed as the best quality level of Parkinson's treatment. Levodopa works by crossing point the blood-mind limit, the perplexing meshwork of fine veins and cells that channel blood showing up at the frontal cortex, where it is changed over into dopamine. Little segment of levodopa is required to treat appearances. This improvement furthermore lessens the genuine affliction and disgorging consistently experienced as an indication of levodopa. For most patients, levodopa reduces the signs of continuousness, strength and shudder. It is especially feasible for patients that have an insufficiency of unconstrained turn of events and muscle rigidity. This solution, in any case, doesn't stop or moderate the development of the contamination. Levodopa is accessible as a norm (or quick) discharge equation or a long-acting or "controlled-discharge" recipe. Controlled delivery may give a more illustrated-out term of activity by developing the time it takes for the abdominal plot to integrate the medicine. Results may incorporate sickness, spewing, dry mouth and wooziness. Dyskinesias (strange developments) may happen as the portion is expanded. In certain patients, levodopa may create turmoil, mind flights or psychosis.

\section{Bromocriptine:}

Bromocriptine, pergolide, pramipexole and ropinirole are remedies that duplicate the work of substance dispatches in the frontal cortex, causing the neurons to react as they would to dopamine. They can be supported alone or with levodopa and may be used before all else periods of the contamination or figured out how to broaden the term of suitability of levodopa. These medications overall have a bigger number of results than levodopa, so that is considered before experts embrace dopamine agonists to patients. Results may incorporate tiredness, queasiness, retching, dry mouth, wooziness and feeling faint after standing. While these side effects are normal when beginning a dopamine agonist, they generally resolve more than a few days. In certain patients, dopamine agonists may create turmoil, mind flights or psychosis.

\section{Surgery:}

For some patients with Parkinson's, meds are viable for keeping a decent personal satisfaction. As the problem advances, nonetheless, a few patients create changeability in their reaction to treatment, known as "engine variances. During "on" periods, a patient may move effortlessly, frequently with diminished quake and solidness. During "off" periods, patients may have more trouble controlling developments. Off periods may happen only preceding a patient taking their next portion of medicine, and these scenes are designated "wearing off." Uncontrolled squirming developments, called dyskinesias, may result. These issues can generally be dealt with changes in drugs. In view of the sort and seriousness of indications, the disintegration of a patient's personal satisfaction, and a patient's general wellbeing, medical procedure might be the subsequent stage. The advantages of a medical procedure ought to consistently be weighed cautiously against its dangers, thinking about the patient's manifestations and in general wellbeing.

\section{DATA SET USED}

The dataset we will be dealing with was acquired from UCI AI vault. We are anticipating demonstrating that by testing the voice and the winding trial of the patient is a noticeable method to analyze them for the illness. The current determination will be significant as tests should be possible with most extreme exactness saving time, cost and energy. The task will endeavor to demonstrate that mind movement and voice information from the patients can help identify/analyze Parkinson's illness. As there is a connection between the two. I will endeavor to run different AI classifiers like KNN, matrix looking, strategic relapse, SVM, choice tree, arbitrary woodland and neural organizations on the information to arrive at high consistency with a less runtime. By playing out a grouping on this information, I desire to demonstrate that vocalization tests are an appropriate method to analyze a patient for this infection.

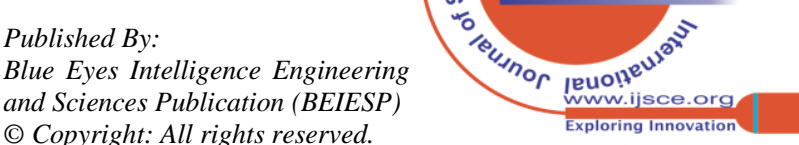


The current determination is important as the test can be directed at home without the requirement for the patient to visit a clinic - saving time, cost, and energy.

\section{METHODOLOGY AND ALGORITHM USED}

Supervised learning gives you a useful asset to characterize and deal with information utilizing machine language. With administered learning you utilize marked information, which is an informational index that has been arranged, to induce a learning calculation. The informational collection is utilized as the reason for foreseeing the order of other unlabeled information using AI calculations. There are classifications that go under this space, i.e., Linear Regression, and Classification Techniques.

In the supervised learning (SL) structure, one forms a model by misusing the accessible perceptions through a learning calculation that can catch the data covered up with the information. All in all, managed AI (ML) calculations deduce an info yield connection from a bunch of information that comprises of instances of this connection. This stage is typically called the "preparation stage." Because of the fast development of the quantity of tests, of the dimensionality of the information and yield space, and of the assortment and structure of the information that are moved, put away, and handled, the traditional ML techniques began to show their cutoff points. In particular, the issue of tuning the presentation of the calculations-specifically, model determination (MS) — and thoroughly assessing their exhibition.

Supervised learning is essentially an equivalent for arrangement. The management in the taking in comes from the marked models in the preparation dataset. For instance, in the postal code acknowledgment issue, a bunch of manually written postal code pictures and their comparing machine-decipherable interpretations are utilized as the preparation models, which administer the learning of the order model. In this paper, generally regulated learning is accustomed to carry some helpful bits of knowledge with incredible exactness and accuracy.

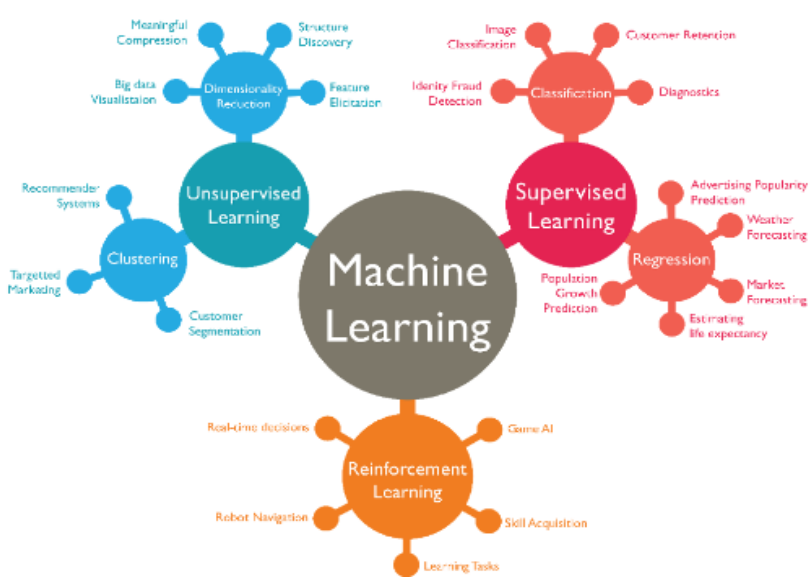

Fig.1. Machine Learning Architecture

\section{A. Logistic Regression}

Logistic regression is a strategy assessment used to give out experiences to a discrete arrangement of classes. Not at all like straight fall away from the faith which yields consistent, not really settled to apostatize changes its yield utilizing the fundamental sigmoid capacity to restore likelihood respect which would then have the alternative to be needed to something like two discrete classes.

Logistic Regression could assist with anticipating whether the understudy passed or failed. Vital backslide figures are discrete (simply unequivocal characteristics or classes are allowed). We can in like manner see probability scores crucial the model's groupings.

Logistic backslide computation furthermore uses an immediate condition with free pointers to predict a value. The expected worth can be any place between unfavorable boundlessness to positive propagation. We need the yield of the assessment to be a class variable, i.e., 0-no, 1-yes. Hence, we are crushing the yield of the straight condition into a degree of $[0,1]$. To pound the normal a catalyst some spot in the extent of 0 and 1 , we utilize as far as possible.

$$
\begin{gathered}
z=\theta_{0}+\theta_{1} \cdot x_{1}+\theta \cdot x_{2}+\cdots \\
g(z)=\frac{1}{1+e^{-z}} \\
h=g(z)=\frac{1}{1+e^{-z}}
\end{gathered}
$$

We take the output(z) of the direct condition and offer it to the capacity $\mathrm{g}(\mathrm{x})$ which restores a crushed worth $\mathrm{h}$, the worth $\mathrm{h}$ will lie in the scope of 0 to 1

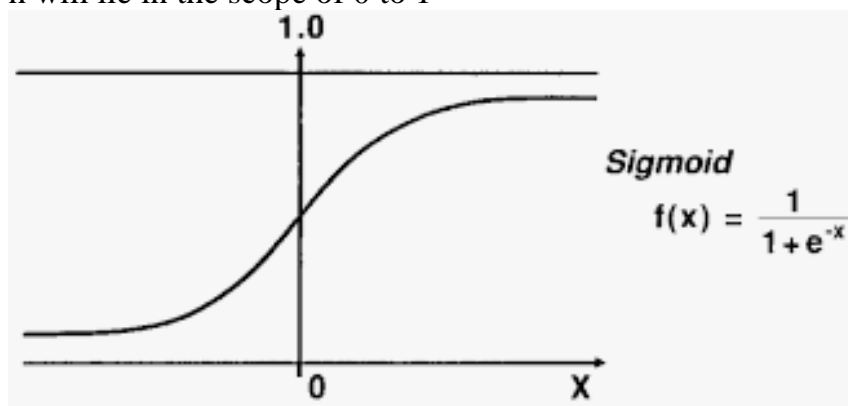

Fig.2. Sigmoid function representation

\section{B. K-Nearest Neighbor}

KNN can be utilized for both characterization and relapse prescient issues. The calculation fairs across all boundaries of contemplations. It is generally utilized for its simplicity of translation and low figuring time.

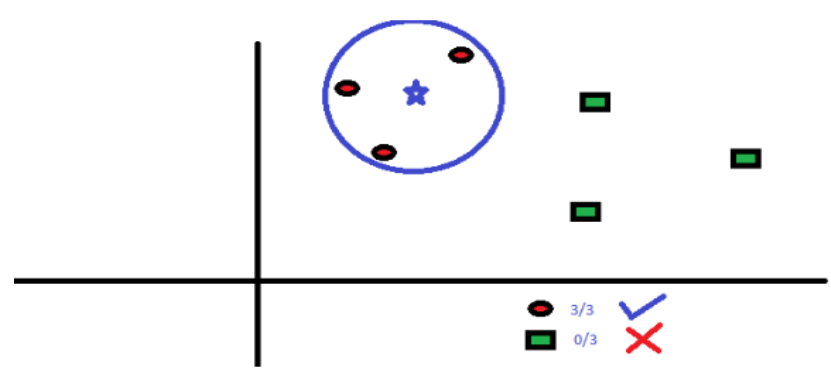

Fig.3. Nearest Neighbor Representation

The "K" is KNN calculation is the closest neighbor we wish to take the vote from. Expect to be $K=3$. Subsequently, we will at present make a float with BS as the middle relatively as broad as to encase just three server farms around the plane. The three nearest focuses to BS are all RC. Along these lines, with a decent conviction level, 


\section{Application of AI for Analysis of Parkinson's Disease}

we can say that the BS ought to have a spot with the class RC. Here, the decision wound up being amazingly clear as all of the three votes from the nearest neighbor went to RC. The decision of the cutoff $\mathrm{K}$ is sincere in this assessment. Then, at that point, we will get a handle on what are the elements to be considered to wrap up the best $\mathrm{K}$. If we see the last model, given that all the 6 getting ready insights stay consistent, with a given $\mathrm{K}$ worth we can make cutoff points of each class. These cutoff points will separate RC from GS. Coming up next are as far as possible separating the two classes with different assessments.
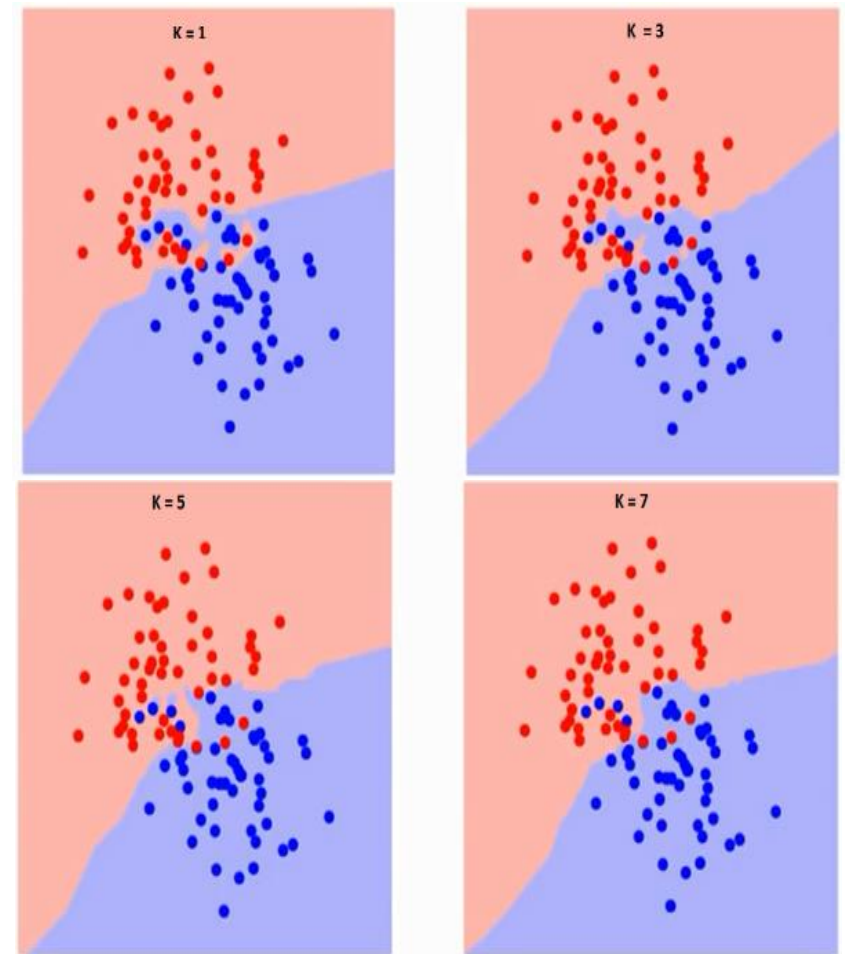

Fig.4. Isolating the two classes with various estimations of K.

Following is the curve generated for $\mathrm{K}$.

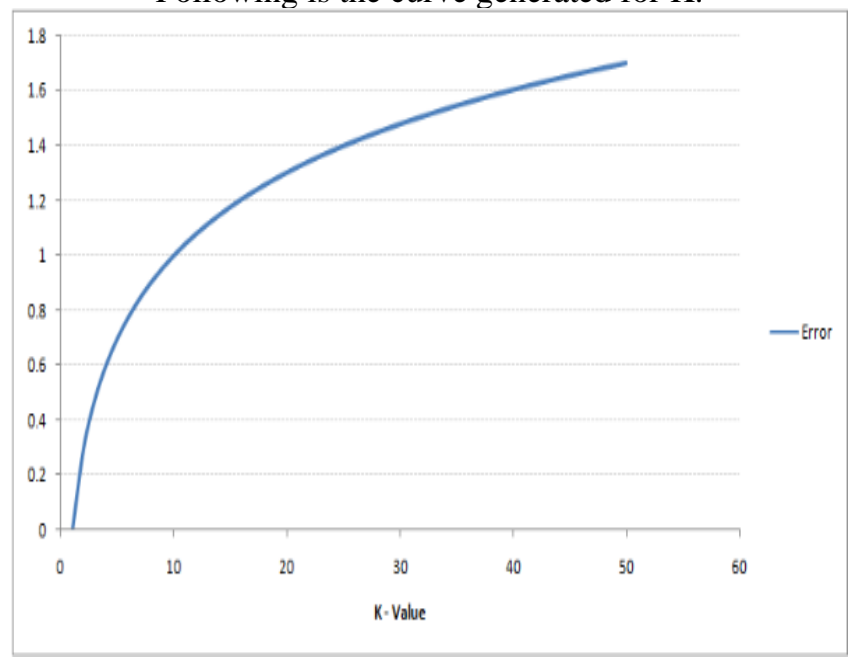

Fig.5. Curve for the training error rate using $\mathrm{K}$

As ought to be self-evident, the mix-up rate at $\mathrm{K}=1$ is reliably zero for the arrangement test. This is because the closest feature any planning data point is itself. In this manner the assumption is reliably precise with $K=1$. In case the endorsement screws up curve would have been near; our choice of $\mathrm{K}$ would have been 1 . Following is the endorsement botch twist with the fluctuating assessments of $\mathrm{K}$.

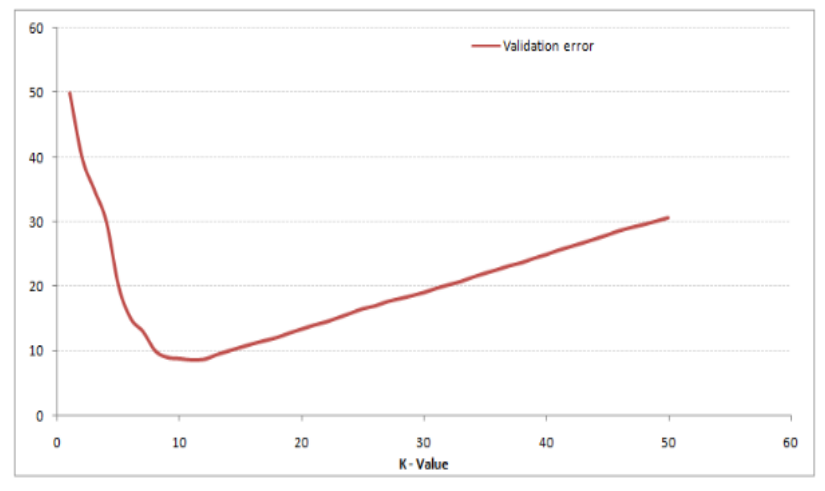

Fig.6. False Curve with wrong assumptions of $\mathbf{K}$

This makes the story more comprehended. At $K=1$, we were overfitting the cutoff points. From this time forward, the bumble rate at first decreases and shows up at any rate. After the minima point, it by then additions with extending $\mathrm{K}$. To get the best assessment of $\mathrm{K}$, you can disengage the planning and endorsement from the hidden dataset.

\section{Decision Trees}

Decision Trees are a non-parametric skillful learning method used for request and backslide. The goal is to make a model that predicts the valuation of a target variable by taking in direct decision strategies deduced from the data features.

For instance, in the model under, choice trees acquire from data to harsh a sine twist with a lot of if else decision rules. The more significant the tree, the more awesome the decision guidelines and the fitter the model.

Some advantages of decision trees are:

- Simple to decipher and to comprehend. Trees can be proposed.

- Requires little information planning. Different procedures regularly require information standardization, faker factors should be made and clear qualities to be taken out. Note anyway that this module doesn't uphold missing qualities.

- The cost of utilizing the tree (i.e., foreseeing information) is logarithmic in the quantity of information focuses used to prepare the tree.

- Able to deal with both mathematical and straight-out information. Different methods are typically worked in dissecting datasets that have just one sort of factor.

- Able to deal with multi-yield issues. Performs well regardless of whether its suppositions are fairly abused by the genuine model from which the information were created.

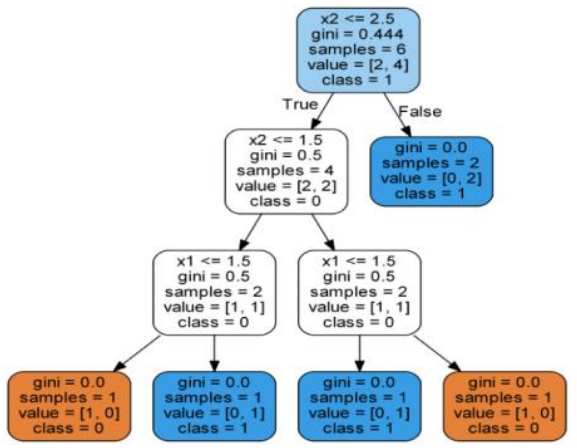

Fig.6. Sample Decision Tree

Published By:

Blue Eyes Intelligence Engineering and Sciences Publication (BEIESP)

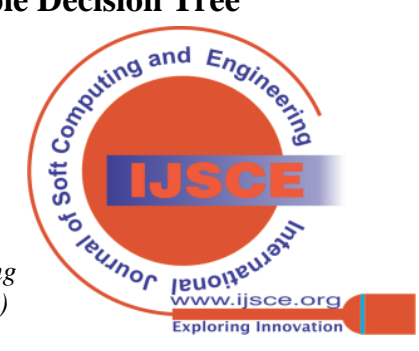




\section{Random Forest}

The Random Forest combines hundreds or thousands of decision trees, plans everybody on an imperceptibly exceptional course of action of discernments, separating center points in each tree contemplating a set number of the features. The last assumptions for the self-assertive forest area are made by averaging the gauges of each individual tree. It is an outfit model made of various decision trees using bootstrapping, sporadic subsets of features, and ordinary majority rule to make assumptions. This is a delineation of a sacking outfit.

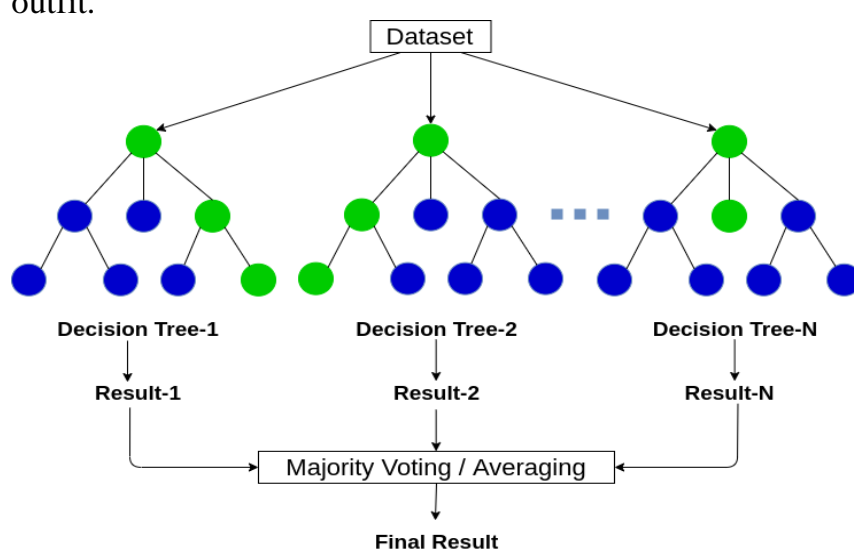

Fig.7. Random Forest Classifier

\section{E. Extremely Randomized Trees Classifier}

It is a kind of get-together learning procedure that adds up to the outcomes of different de-related decision trees accumulated in a "forest area" to yield its gathering result. In thought, it is essentially equivalent to a Random Forest Classifier and simply differentiates from it in the method of improvement of the decision trees in the woodlands. Each Decision Tree in the Extra Trees Forest is worked from the first planning test. By then, at each test center, each tree is given a self-assertive illustration of $\mathrm{k}$ features from the rundown of abilities from which each decision tree ought to pick the best segment to part the data subject to some mathematical standards (conventionally the Gini Index

\section{IMPLEMENTATION AND RESULTS}

The following are the results from the dataset 1 . After performing EDA here some insights that I found about the patients through some interesting visualization.

- The below figure shows the box plot of the frequency variation. All the three variations have outliers. Decision trees can handle outliers. It is very unlikely that a decision tree will create a leaf to isolate them.

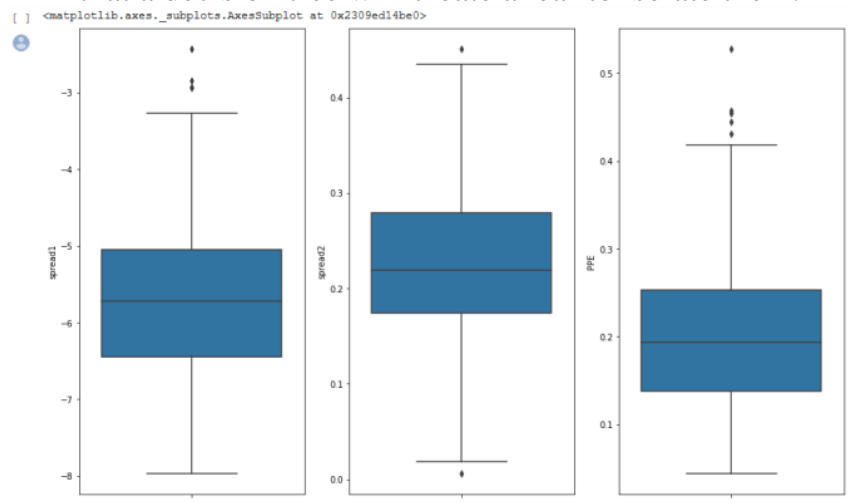

- Next, the measures of vocal fundamental frequency are shown above. There is a positive skewness for minimum vocal fundamental frequency with more high values between $75 \mathrm{~Hz}$ and $125 \mathrm{Hhz}$. The average vocal frequency is almost normally distributed with more values ranging $115 \mathrm{~Hz}$ and $130 \mathrm{~Hz}$. The high vocal frequency does not have any skewness, but some ranges of values are at the rightmost tail.

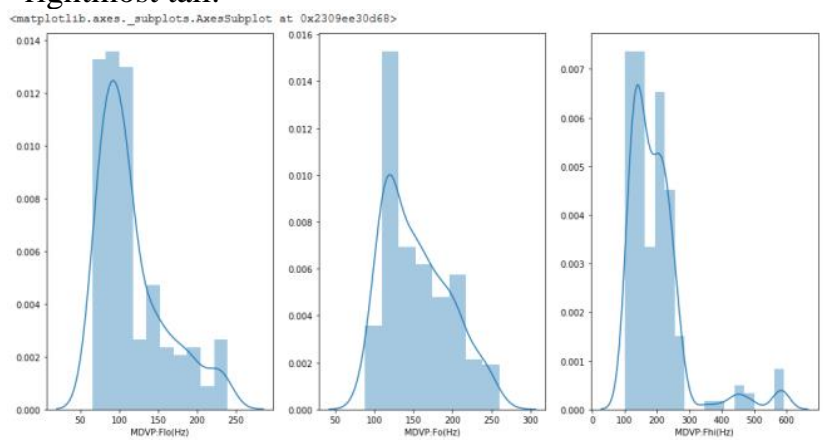

- Here, the measure of the tonal component of frequency is shown above. The value NHR is right skewed for there are so many observations in the area, but they seem to be with very minimal values. The maximum number of observations is between 0 and 0.04 . The value HNR looks like normally distributed, but on a first look there seems to be a slight negative skewness.
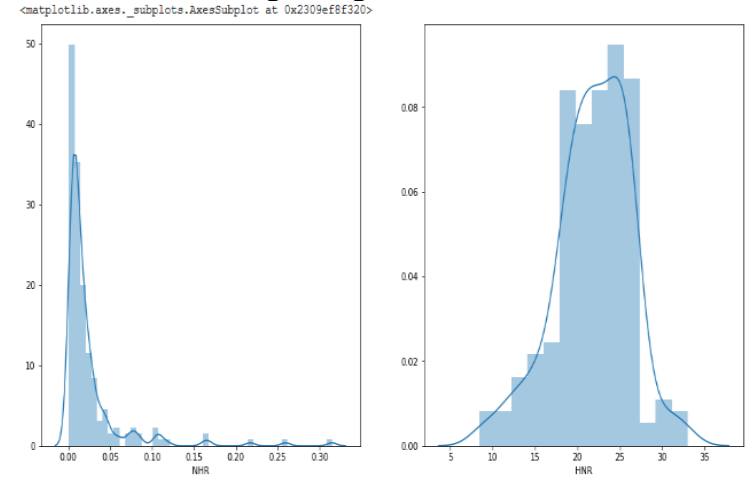

- For all the below graphs, we can observe that the measure of variation in amplitude is positively skewed.
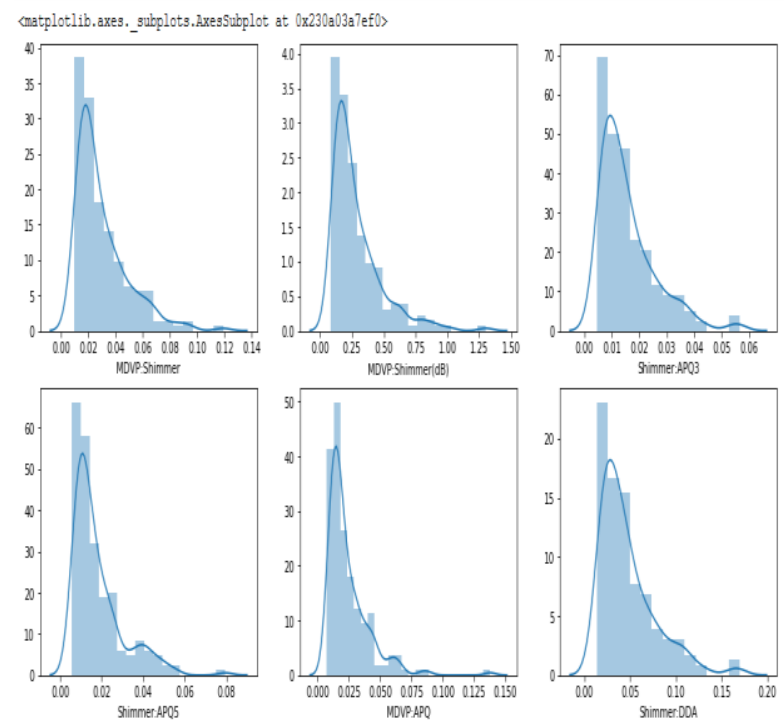

Published By:

Blue Eyes Intelligence Engineering and Sciences Publication (BEIESP) 37 (C) Copyright: All rights reserved.

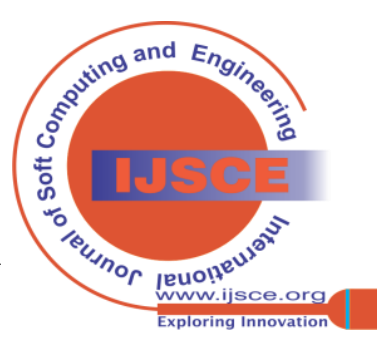




\section{Application of AI for Analysis of Parkinson's Disease}

- Spread1 is normally distributed between people who have - 8.5 and -7.5 are more and they are normal. People whose PD and who are normal. People who have spread1 between spread1 is between -6.5 and -5 are having PD.

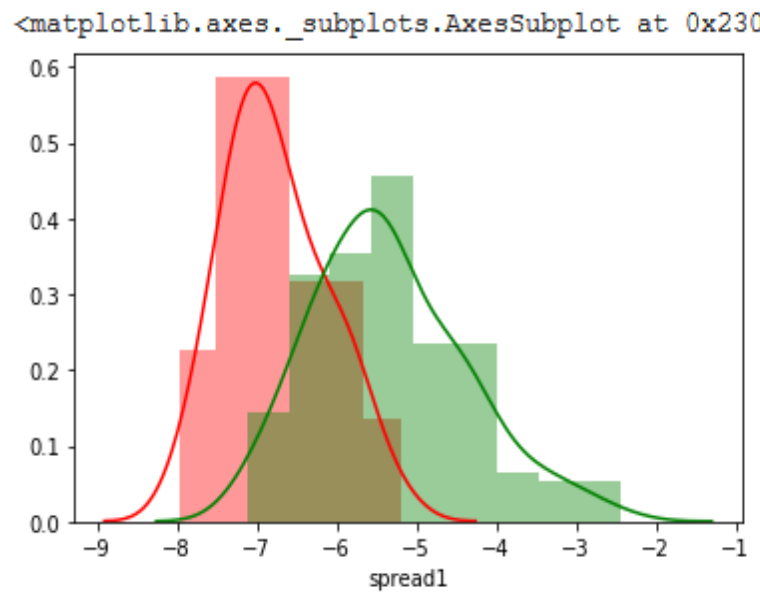

Fig. 7. Graphical spread between Normal people and PD people

- People who have PD (status equal to one) have higher levels of Noise to Harmonic ratio. Also, looking into the HNR ratio people who have PD have lower levels in the same.

-When we look at the relationship between status and MDVP:Fo(Hz) we can see the median value is around $199 \mathrm{~Hz}$ for people who are normal. For people who are affected with Parkinson's the median value comes around $145 \mathrm{~Hz}$.

- People who are suffering for PD tend to have higher jitter \%. It seems if the values go above 0.15 we can confirm the patient is having PD. The variation of fundamental frequency is in a low range for people who are normal.

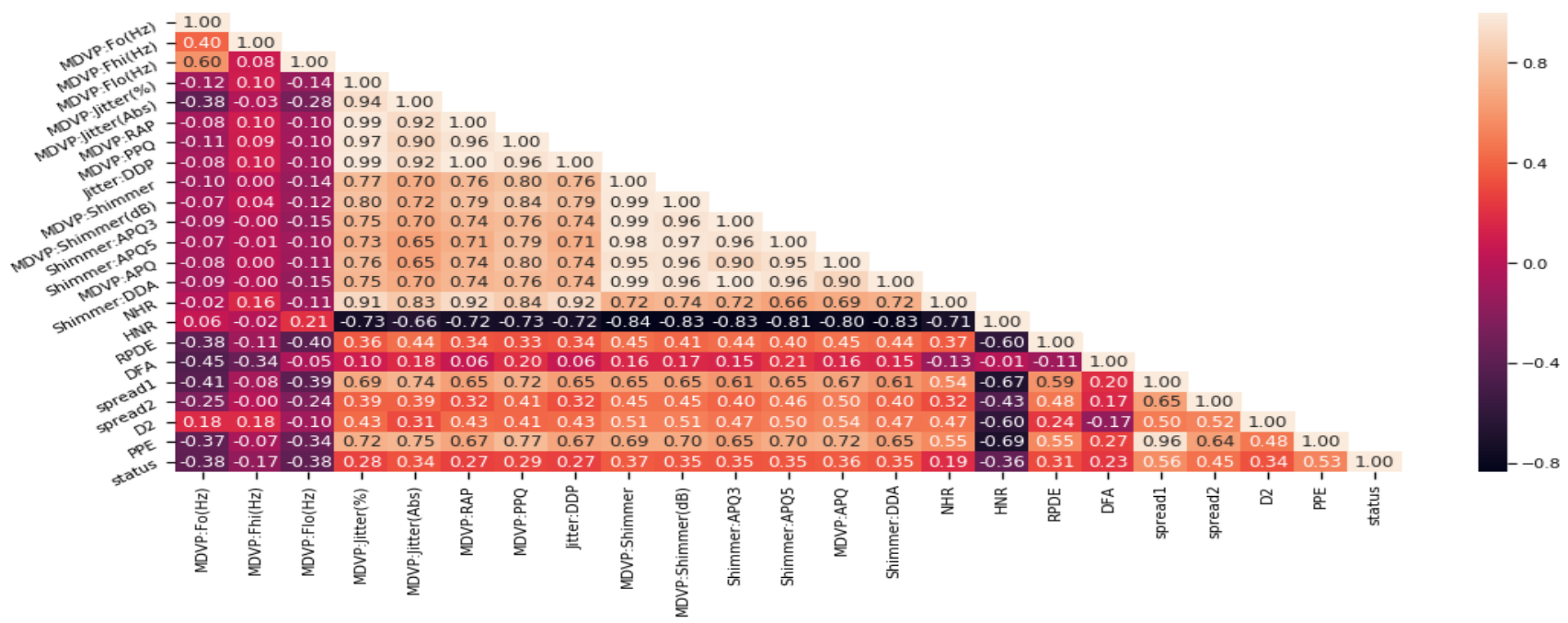

Fig.8. Correlation Map Plot

Random forest classifier is used to formulate the features that are there in the dataset, here is the feature importance that model is showing.

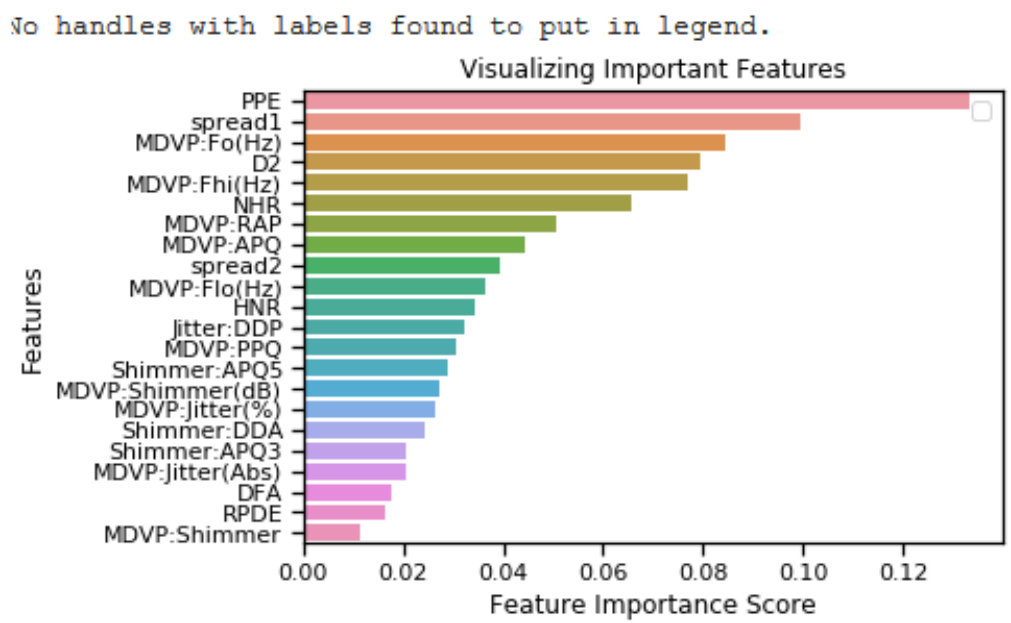

Fig.9. Feature importance using RF Classifier.

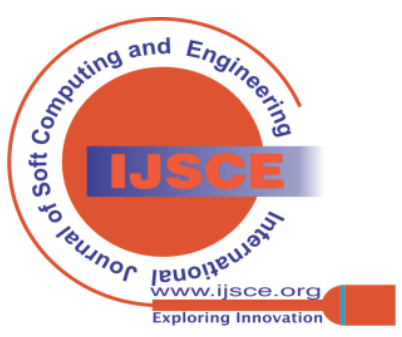




\section{CONCLUSION}

To the most amazing aspect our insight, the current investigation is the principal thorough survey that included outcomes from all examinations that applied AI strategies to the finding of PD. Here, we introduced remembered reads for a significant level rundown, giving admittance to data including (a) AI techniques that have been utilized in the conclusion of PD and related results, (b) kinds of clinical, conduct and biometric information that could be utilized for delivering more precise.

\section{REFERENCES}

1. G. O. Young, "Synthetic structure of industrial plastics (Book style with D. Hirtz, D. Thurman, K. Gwinn-Hardy, M. Mohamed, A. Chaudhuri, and R. Zalutsky, "How common are the "common" neurologic disorders?" Neurology, vol. 68, no. 5, pp. 326-337, 2007.

2. E. R. Dorsey and B. R. Bloem, "The parkinson pandemic-a call to action," JAMA neurology, vol. 75, no. 1, pp. 9-10, 2018.

3. [3] J. Jankovic, "Parkinson's disease: clinical features and diagnosis," Journal of Neurology, Neurosurgery \& Psychiatry, vol. 79, no. 4, pp. 368-376, 2008.

4. W. Poewe, "The natural history of parkinson's disease," Journal of neurology, vol. 253, no. 7, pp. vii2-vii6, 2006.

5. K. R. Chaudhuri, D. G. Healy, and A. H. Schapira, "Non-motor symptoms of parkinson's disease: diagnosis and management," The Lancet Neurology, vol. 5, no. 3, pp. 235-245, 2006.

6. M. M. Goldenberg, "Medical management of parkinson's disease," Pharmacy and Therapeutics, vol. 33, no. 10, p. 590, 2008

7. R. L. Brey, "Muhammad ali's message: Keep moving forward," Neurology Now, vol. 2, no. 2, p. 8, 2006.

8. M. H. Polymeropoulos, C. Lavedan, E. Leroy, S. E. Ide, A. Dehejia, A Dutra, B. Pike, H. Root, J. Rubenstein, R. Boyer, et al., "Mutation in the $\alpha$-synuclein gene identified in families with parkinson's disease," science, vol. 276, no. 5321, pp. 2045-2047, 1997.

9. S. Fahn, "Description of parkinson's disease as a clinical syndrome," Annals of the New York Academy of Sciences, vol. 991, no. 1, pp. 1-14 2003.

10. S. Arora, V. Venkataraman, A. Zhan, S. Donohue, K. Biglan, E. Dorsey, and M. Little, "Detecting and monitoring the symptoms of parkinson's disease using smartphones: A pilot study," Parkinsonism \& related disorders, vol. 21, no. 6, pp. 650-653, 2015.

11. M. A. Little, P. E. McSharry, E. J. Hunter, J. Spielman, L. O. Ramig, et al., "Suitability of dysphonia measurements for telemonitoring of parkinson's disease," IEEE transactions on biomedical engineering, vol. 56, no. 4, pp. 1015-1022, 2009.

12. B. E. Sakar, M. E. Isenkul, C. O. Sakar, A. Sertbas, F. Gurgen, S. Delil, H. Apaydin, and O. Kursun, "Collection and analysis of a parkinson speech dataset with multiple types of sound recordings," IEEE Journal of Biomedical and Health Informatics, vol. 17, no. 4, pp. 828-834, 2013.

13. B. M. Bot, C. Suver, E. C. Neto, M. Kellen, A. Klein, C. Bare, M. Doerr, A. Pratap, J. Wilbanks, E. R. Dorsey, et al., "The mpower study, parkinson disease mobile data collected using researchkit," Scientific data, vol. 3, p. 160011, 2016

14. M. Little, "Ubiquitous, inexpensive non-invasive technologies for objective detection and monitoring of parkinson's symptoms," 2013.

15. F. L. Pagan, "Improving outcomes through early diagnosis of parkinson's disease." The American journal of managed care, vol. 18, no. 7 Suppl, pp. S176-82, 2012.

16. Tysnes, O.B.; Storstein, A. Epidemiology of Parkinson's disease. J Neural Transm. 2017, 124, 901-905.

17. Parkinson's Disease Detection from Drawing Movements Using Convolutional Neural Networks

18. Manuel Gil-Martín , Juan Manuel Montero and Rubén San-Segundo Rizzo, G.; Copetti, M.; Arcuti, S.; Martino, D.; Fontana, A.; Logroscino, G. Accuracy of clinical diagnosis of Parkinson disease: A systematic review and meta-analysis.

19. Distinguishing Different Stages of Parkinson's Disease Using Composite Index of Speed and Pen-Pressure of Sketching a Spiral.Poonam Zham1*, imageDinesh K. Kumar1, imagePeter Dabnichki1, imageSridhar Poosapadi Arjunan1 and imageSanjay Raghav1,2

20. Feature visualization and classification for the discrimination between individuals with Parkinson's disease under levodopa and DBS treatments. Alessandro R. P. Machado, corresponding author1 Hudson Capanema Zaidan,1 Ana Paula Souza Paixão,1 Guilherme Lopes Cavalheiro,1 Fábio Henrique Monteiro Oliveira,1 João Areis Ferreira
Barbosa Júnior,1 Kheline Naves,1 Adriano Alves Pereira,1 Janser Moura Pereira,2 Nader Pouratian,3 Xiaoyi Zhuo,3 Andrew O'Keeffe,3 Justin Sharim,3 Yvette Bordelon,4 Laurice Yang,5 Marcus Fraga Vieira, 6 and Adriano O. Andrade1

21. Parkinson's Dataset Study: IEEE Journal of Biomedical and Health Informatics. (n.d.). Retrieved June 02, 2016, from http://ieeexplore.ieee.org/xpl/RecentIssue.jsp?punumber=6221020

22. 'Exploiting Nonlinear Recurrence and Fractal Scaling Properties for Voice Disorder Detection', Little MA, McSharry PE, Roberts SJ, Costello DAE, Moroz IM. BioMedical Engineering OnLine 2007, 6:23 (26 June 2007)

\section{AUTHORS PROFILE}

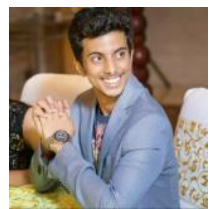

Harsh Pandey, is an IT professional currently working in Accenture. His interest includes Framework development, Network Security and he has a keen eye on application of Machine Learning to various real time systems including IDS and key frame encryptions. An alumni of Delhi Public School R.k. Puram, he has completed his Bachelor's in Technology in "Information Technology" from Manipal University Jaipur in 2020

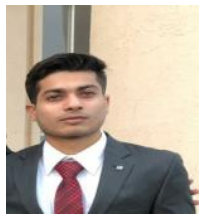

Arjun Shivnani, is an IT professional working at Bluprints. His interests include Software Testing(Manual and Automation) using selenium. His specialization is automating machine learning algorithm for effective programming which reduce redundancy. An alumni of Delhi Public School R.k. Puram, he has completed his Bachelor's in Technology in "Computers And Communication" from Manipal University Jaipur in 2020

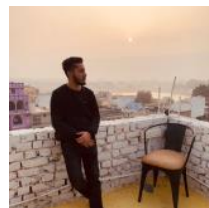

Aditya Pratap Singh, a student of B.tech at Manipal University Jaipur. His goal is to solve complex real-world problems via big data. He is passionate about Computer vision, Software Engineering, and machine learning techniques.

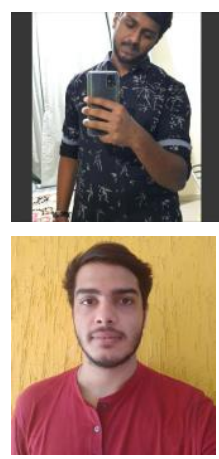

Pauras, is especially interested in the research of Natural Language Processing to improve speech integrated systems. He would like to pursue post graduate studies in Computer Science to broaden my knowledge in this field.

Aryamaan Singh Chauhan, is a graduate from Manipal University, Jaipur in B.Tech Information Technology. He is an Alumni of Army Public School Dhaula Kuan, New Delhi. His field of interest are Machine learning and Natural Language Processing.
Published By:

Blue Eyes Intelligence Engineering and Sciences Publication (BEIESP) 39 (C) Copyright: All rights reserved.

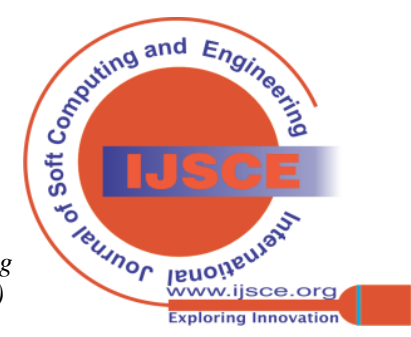

